\title{
Mitogen-activated protein kinase phosphatase-1 expression in macrophages is controlled by lymphocytes during macrophage activation
}

\author{
CHONG LUO, XIQIANG YANG, LAN YAO, LIPING JIANG, WEI LIU, XIN LI and LIJIA WANG \\ Ministry of Education Key Laboratory of Child Development and Disorders, \\ Children's Hospital of Chongqing Medical University, Chongqing 400014, P.R. China
}

Received June 14, 2011; Accepted August 11, 2011

DOI: $10.3892 / \mathrm{ijmm} .2011 .799$

\begin{abstract}
The viewpoints on the control of innate immune cells by the adaptive immune system during sepsis remain controversial. Mitogen-activated protein kinase phosphatase-1 (MKP-1) is essential to the negative control of innate immunity and suppresses the activation of macrophages by inhibiting activated mitogen-activated protein kinase (MAPK). The purpose of the current study was to observe inflammatory response and macrophage activation in mice with severe combined immunodeficiency (SCID) with endotoxemia and to determine the role of MKP-1 in the control of macrophage activation by the adaptive immune system. Endotoxemia was induced in wild-type and SCID mice by an intraperitoneal injection of lipopolysaccharide (LPS), and all of the SCID mice died. SCID mice produced more inflammatory cytokines than BALB/c mice systemically and locally. TNF- $\alpha$ mRNA expression was higher and MKP-1 mRNA expression was lower in peritoneal macrophages (PMa) from SCID mice compared to PMa from wild-type mice after and even before LPS injection. Thioglycollate-stimulated PMa from wild-type mice were stimulated with LPS in vitro in the presence or absence of pan-T cells. The levels of TNF- $\alpha$ and IL- 6 were higher in the supernatants from PMa cultured alone compared to PMa co-cultured with pan-T cells, and PMa MKP-1 mRNA and protein expression were higher when PMa were co-cultured with pan-T cells. Therefore, pan-T cells can up-regulate MKP-1 expression in macrophages and inhibit the secretion of inflammatory cytokines secretion by macrophages. In SCID mice, lymphocyte deficiency, especially $\mathrm{T}$ cell deficiency, causes
\end{abstract}

Correspondence to: Professor Xiqiang Yang, Ministry of Education Key Laboratory of Child Development and Disorders, Children's Hospital of Chongqing Medical University, 136 Zhongshan No. 2 Road, Yu Zhong District, Chongqing 400014, P.R. China

E-mail: xiqyang@163.com

Key words: macrophages, endotoxemia, lymphocyte deficiency, mitogen-activated protein kinase phosphatase-1 insufficient MKP-1 expression in macrophages, which can be responsible for the severe inflammation and bad prognosis of septic SCID mice. MKP-1 plays an important role in the control of macrophage activation by the adaptive immune system.

\section{Introduction}

Sepsis, a syndrome with a relatively high mortality rate, is a severe systemic inflammatory response syndrome (SIRS) that can be triggered by bacterial, virus, fungal or parasitic infection and is often the result of events such as surgery, trauma, burns, illness, cancer or pneumonia (1). Syndromes with high mortality rates, such as infection-associated hemophilia syndrome (IAHS) and severe acute respiratory syndrome (SARS) which was highly prevalent in 2003 in China, and severe H1N1 infection which individuals suffered from in 2009 worldwide are also characterized by uncontrolled pathophysiological inflammation (2-4). The abnormal activation of innate immune cells is the basis of uncontrolled inflammation and results in the occurrence of severe syndromes (5-7). However, the question of what contributes to the abnormal activation of innate immune cells in these syndromes remains unclear. Recent research has indicated that the interaction between innate immune cells and adaptive immune cells is complex. Our previous study (8) showed that after respiratory syncytial virus infection, a more severe inflammatory injury was found in the lungs of $\mathrm{T}$ cell-deficient nude mice than in wild-type mice. Such result suggested that $\mathrm{T}$ cells may inhibit inflammation and innate immunity, and Kim et al (9) showed that lymphocytes can suppress the activation of innate immune cells. Therefore, we speculate that abnormal activation of innate immune cells in sepsis may be related to differentially defects of lymphocytes.

When faced with foreign pathogens, innate immune cells become activated through recognition of PAMPs (pathogenassociated molecular patterns) by PRRs (pattern-recognition receptors). Then, a cascade of signal transduction molecules starts, leading to MAPK activation and the production of inflammatory cytokines. In mammalian cells, mitogen-activated protein kinase phosphatases (MKP) are essential to the negative control of innate immunity and suppress the activation of innate immune cells by inhibiting activated MAPK (10). MKP can 
dephosphorylate the tyrosine residues of activated MAPK, thus reducing production of inflammatory cytokines (10). MKP-1 is an important family member of MKP (11). After lipopolysaccharide (LPS) injection, MKp- $1^{-/-}$mice secrete much more TNF- $\alpha$, IL- 6 and IL-10 than wild-type mice and are more susceptible to septic shock and multiple organ dysfunction syndrome (MODS) (12). In Gram-positive and Gram-negative bacterial infection, MKP-1 prevents the occurrence of septic shock and MODS by limiting the strength and duration of inflammatory signals (13). Therefore, the role of MKP-1 in the control of innate immune cells by the adaptive immune systems needs further study.

In this study, endotoxemia was induced in SCID mice which lack mature $\mathrm{T}$ and $\mathrm{B}$ cells by an intraperitoneal injection of LPS to observe the effect of lymphocyte deficiency on inflammatory response and macrophage activation. Thioglycollate-stimulated peritoneal macrophages (PMa) were stimulated by LPS in vitro in the presence or absence of pan-T cells, allowing for the investigation of the effect of pan-T cells on macrophage activation and the role of MKP-1 in the control of macrophage activation by the adaptive immune system.

\section{Materials and methods}

LPS-induced endotoxemia. Specific pathogen-free, 6- to 8-week-old, 18-22-g, female BALB/c mice and SCID mice (BALB/c background) were purchased from the Academy of Medical Sciences of China and were housed in the Animal Care Facility Unit (Chongqing Children's Hospital, Chongqing Medical University). The mice were kept on a 12-h light/dark cycle. The mice were injected with LPS (E. coli, serotype O111:B4, $1.5 \mathrm{mg} / \mathrm{kg}$ ) intraperitoneally to induce endotoxemia. The Animal Use Committee at Chongqing Children's Hospital, Chongqing Medical University approved all of the experiments conducted in this study.

Peritoneal fluid and isolation of PMa. The mice were sacrificed before LPS injection (control) and at 3,6 and $12 \mathrm{~h}$ after LPS injection (eight mice per group per time point). Ice-cold PBS (1 ml) was injected intraperitoneally. After massaging the mouse abdomen for $5 \mathrm{~min}$, the peritoneal lavage fluid were collected $(\sim 0.75 \mathrm{ml})$ and centrifuged at $200 \mathrm{x} \mathrm{g}$ for $5 \mathrm{~min}$. The supernatants were stored at $-20^{\circ} \mathrm{C}$ for subsequent cytokine assays. Peritoneal exudate cells were resuspended in RPMI-1640 with $10 \%$ fetal bovine serum (FBS) and cultured in 6-well dishes for $30 \mathrm{~min}$ at $37^{\circ} \mathrm{C}$. The dishes were then washed twice with PBS warmed to $37^{\circ} \mathrm{C}$. Adherent cells were recovered from the dishes using a rubber scraper. The purity of the F4/80(+) cells was $85 \%$ or higher, as tested by FACS using a FACSVantage, flow cytometer, in eight independent experiments. Thioglycollate-stimulated PMa were prepared by injecting 3\% thioglycollate medium into BALB/c mice intraperitoneally. Four days later, the mice were sacrificed. PMa were collected as previously described, and the purity of the F4/80(+) cells was $90 \%$ or higher, as tested by FACS, in eight independent experiments.

Isolation of pan-T cells. Spleens from control BALB/c mice were made into a single-cell suspension manually. Lymphocytes separation medium (TBD, China) was used to separate lymphocytes from the single-cell suspension and to remove the dead cells. The Pan-T cell isolation kit II (Miltenyi, German) was used for MACS. Lymphocytes were incubated with Biotin-Antibody Cocktail at $4^{\circ} \mathrm{C}$ for $10 \mathrm{~min}$ and then incubated with Anti-Biotin microbeads at $4^{\circ} \mathrm{C}$ for an additional $15 \mathrm{~min}$. The cells were washed and centrifuged at $300 \mathrm{x} \mathrm{g}$ for $10 \mathrm{~min}$. Then the cells were resuspended in cold buffer. Pan-T cells were isolated using midi-magnetic separation with LS columns. The purity of the CD3(+) cells was $90 \%$ or higher, as tested by FACS.

Cell culture and LPS stimulation in vitro. Thioglycollatestimulated PMa were incubated in the presence or absence of pan-T cells in a $5 \% \mathrm{CO}_{2}$ incubator at $37^{\circ} \mathrm{C}$ for $18 \mathrm{~h}$, and the ratio of PMa to pan-T cells was 1:1. The cells were subsequently stimulated with $100 \mathrm{ng} / \mathrm{ml}$ of LPS. Supernatants were collected for subsequent cytokine assays, and PMa were collected to test for MKP-1 mRNA and protein expression.

RNA extraction and real-time RT PCR. Total cellular RNA was extracted from PMa using the RNA extraction kit (Tiangen, China) according to the manufacturer's recommendations and was reverse transcribed into cDNA using random hexamers and reverse transcriptase (Takara, Japan). The PCR reaction was performed with $2 \mu \mathrm{l}$ of cDNA, $0.1 \mu \mathrm{M}$ of each forward and reverse primers and $12.5 \mu \mathrm{l}$ of SYBR Green PCR Master Mix Reagent (Takara) in a Biorad cycler (USA). The thermal profile for the real-time PCR (RT-PCR) was $95^{\circ} \mathrm{C}$ for $30 \mathrm{sec}$, followed by 40 cycles of $95^{\circ} \mathrm{C}$ for $5 \mathrm{sec}$ and $52^{\circ} \mathrm{C}$ (IL-10) $/ 54^{\circ} \mathrm{C}$ (MKP-1) $/ 60^{\circ} \mathrm{C}$ (TNF- $\alpha$ and $\beta$-actin) for $30 \mathrm{sec}$. Gene expression was expressed as fold change relative to the $\beta$-actin level and was calculated using the $2^{-\Delta \Delta \mathrm{Ct}}$ method. The melting curve analysis was performed to ensure the specificity of the PCR product. The following mouse primers were used: $\beta$-actin 5'-GATTACTGCTCTGGCTCCTAGC-3' (forward) and 5'-GACTCATCGTACTCCTGCTTGC-3' (reverse); TNF- $\alpha$ 5'-AGACCCTCACACTCAGATCATCTTC-3' (forward) and 5'-TTGGTTGTCTTTGAGATCCATGC-3' (reverse); IL-10 5'-CTCTTACTGACTGGCATGAGGATC-3' (forward) and 5'-AAGGAGTCGGTTAGCAGTATGTTG-3' (reverse); MKP-1 5'-GGGTCACTACCAGTACAAGAGCATC-3' (forward) and 5'-ACAAACACTCTCCCTCCAGCATC-3' (reverse).

Protein extraction and Western blot analysis. Total protein extraction was extracted from PMa using a cell lysis kit. Protein samples were separated by sodium dodecyl sulfate polyacrylamide gel electrophoresis (SDS-PAGE) and were transferred to a polyvinylidene fluoride (PVDF) membrane. After blocking, the membrane was incubated with primary antibodies. Polyclonal antibodies specific for $\beta$-actin and MKP-1 (1:200) were purchased from Santa Cruz Biotechnology (Santa Cruz, CA). Finally, the membrane was incubated with goat anti-rabbit IgG-HRP and the antibodies were detected by an ECL Western blot detection reagent.

Immunofluorescence staining of PMa MKP-1. PMa were allowed to adhere onto 10 -mm-diameter cover glasses and fixed with $4 \%$ paraformaldehyde for $20 \mathrm{~min}$. The cellular and nuclear membranes were broken using $0.3 \%$ Triton. Then the samples were blocked with 5\% BSA for $1 \mathrm{~h}$ and incubated with 


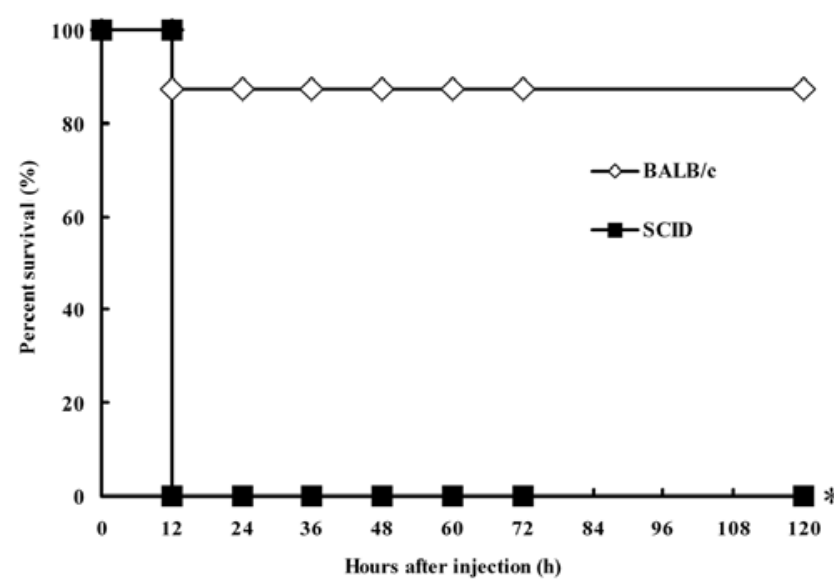

Figure 1. Survival rates of BALB/c mice and SCID mice with endotoxemia induced by LPS. LPS was injected intraperitoneally into BALB/c $(n=8)$ and SCID mice $(n=8)$. The mouse survival data were determined by KaplanMeier test. ${ }^{*} \mathrm{P}<0.05$ as compared to BALB/c mice.

the anti-MKP-1 antibody (1:50) in $1 \% \mathrm{BSA}$ at $4^{\circ} \mathrm{C}$ overnight. After PBS washing, the samples were incubated with goat anti-rabbit IgG-TR in $1 \%$ BSA at room temperature for $1 \mathrm{~h}$. In addition, the nuclei were stained by DAPI at room temperature for $10 \mathrm{~min}$. After washing, the slides were mounted with an anti-fade solution and viewed on a fluorescence microscope.

Measurements of cytokines. Serum samples were collected before treatment and at 3,6, and $12 \mathrm{~h}$ after LPS injection. Serum samples were diluted to 1:10 or 1:5 for Cytometric Bead Array (CBA, BD Bioscience, Cat. No. 552364). Cytokines in the peritoneal fluid and supernatants were quantified using sandwich ELISA kits from R\&D Systems.

Statistical analysis. All data are reported as the mean \pm standard error of mean (SEM). The analysis of variance (ANOVA) was used to compare mean values between normally distributed data. A two-sided P-value of $<0.05$ was considered to be statistically significant. The mouse survival data were determined by Kaplan-Meier test.

\section{Results}

Survival rates and cytokines in serum and peritoneal fluid. After LPS injection, all of the SCID mice died between 12 to $24 \mathrm{~h}$, but only one BALB/c mouse died $(\mathrm{p}<0.05)$ (Fig. 1). The control BALB/c mice and the control SCID mice had similar serum concentrations of the measured cytokines. After LPS injection, the levels of TNF- $\alpha$, IL-10 and IL-6 increased sharply in BALB/c mice and SCID mice. The peak time of TNF- $\alpha$ and IL-10 production was at $3 \mathrm{~h}$ after LPS injection in both mice. As compared to BALB/c mice, SCID mice produced more TNF- $\alpha$ and IL-10 at all time points after LPS injection $(\mathrm{P}<0.05)$ (Fig. 2A). The SCID mice produced more IL-6 (22.32 ng/ml \pm $9.334 \mathrm{ng} / \mathrm{ml}$ vs. $7.479 \mathrm{ng} / \mathrm{ml} \pm 3.579 \mathrm{ng} / \mathrm{ml}, \mathrm{n}=8, \mathrm{p}<0.05)$ than $\mathrm{BALB} / \mathrm{c}$ mice at $12 \mathrm{~h}$ after LPS injection.

There were no differences in TNF- $\alpha$ and IL-10 levels from the peritoneal fluid between control BALB/c and SCID mice. The peak time of TNF- $\alpha$ production in the peritoneal fluid
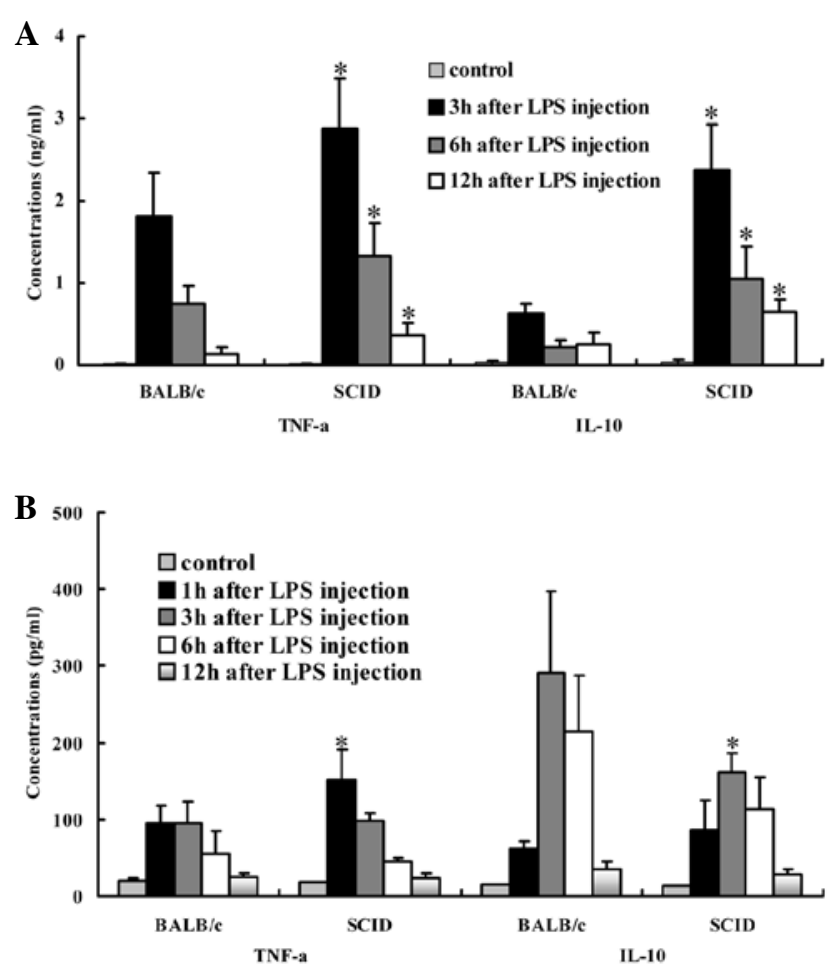

Figure 2. Increased systemic inflammatory response in SCID mice as compared to BALB/c mice after LPS injection. (A) Concentrations of TNF- $\alpha$ and IL-10 in the serum of control and LPS-injected mice were tested by CBA ( $n=7-8$ mice/group). (B) Concentrations of TNF- $\alpha$ and IL-10 in the peritoneal fluid of control and LPS-injected mice were tested by ELISA ( $\mathrm{n}=8$ mice/ group). ${ }^{*} \mathrm{p}<0.05$ as compared to BALB/c mice.

of BALB/c and SCID mice was $1 \mathrm{~h}$ after LPS injection, and SCID mice produced more TNF- $\alpha$ than BALB $/ \mathrm{c}$ mice $(\mathrm{P}<0.05$, Fig. 2B). However, BALB/c mice produced significantly more IL-10 than SCID mice in the peritoneal fluid at $3 \mathrm{~h}$ after LPS injection ( $\mathrm{P}<0.05$, Fig. $2 \mathrm{~B})$.

Effect of lymphocyte deficiency on TNF- $\alpha$ and IL-10 mRNA expression in PMa in vivo. TNF- $\alpha$ mRNA expression was higher and IL-10 mRNA expression was lower in PMa from control SCID mice than PMa from control BALB/c mice. The peak time of TNF- $\alpha$ and IL-10 mRNA expression in PMa from $\mathrm{BALB} / \mathrm{c}$ mice was $1 \mathrm{~h}$ after LPS injection. With the exception of the $1 \mathrm{~h}$-post LPS injection time point, TNF- $\alpha$ mRNA expression in PMa from SCID mice was higher than PMa from $\mathrm{BALB} / \mathrm{c}$ mice at any time point after LPS injection $(\mathrm{P}<0.05)$ (Fig. 3A). IL-10 mRNA expression in PMa from BALB/c mice was higher than PMa from SCID mice at each time point after LPS injection $(\mathrm{P}<0.05)$ (Fig. 3B).

Effect of lymphocyte deficiency on MKP-1 mRNA and protein expression in PMa in vivo. MKP-1 mRNA expression in $\mathrm{PMa}$ from BALB/c and SCID mice had no obvious elevation after LPS injection. MKP-1 mRNA expression in PMa was higher in control BALB/c mice than in control SCID mice, and MKP-1 mRNA expression in PMa from BALB/c mice was higher than in PMa from SCID mice at any time point after LPS injection $(\mathrm{P}<0.05)$ (Fig. 4A). At $1 \mathrm{~h}$ after LPS injection, MKP-1 protein expression was more pronounced in PMa from $\mathrm{BALB} / \mathrm{c}$ mice than SCID mice (Fig. 4B). 

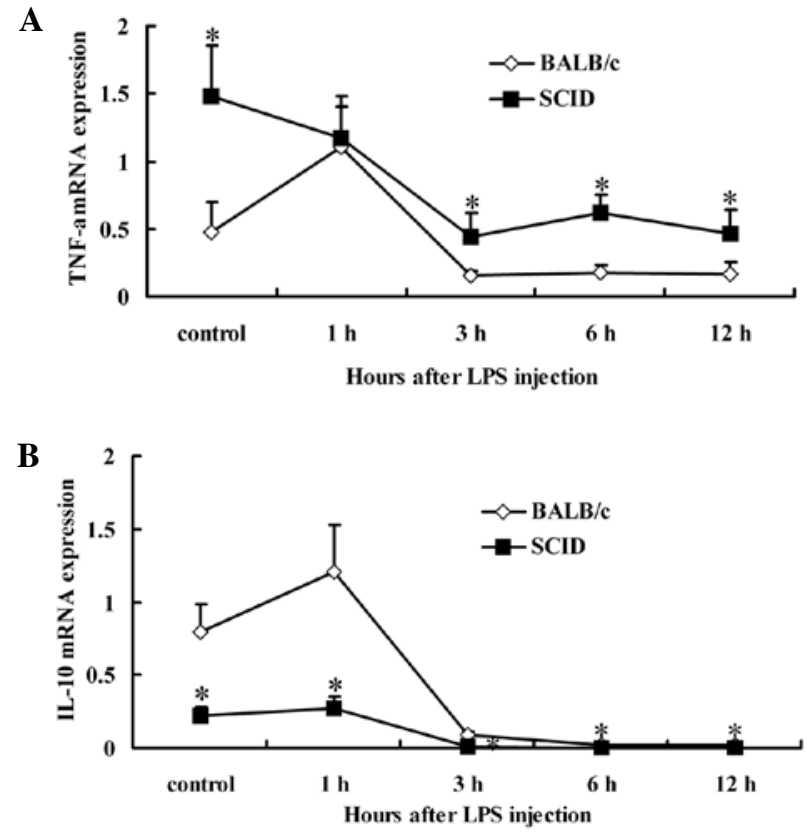

Figure 3. Higher TNF- $\alpha$ expression and lower IL-10 mRNA expression in PMa from SCID mice as compared to PMa from BALB/c mice. (A) TNF- $\alpha$ expression of PMa from control and LPS-injected mice was tested by realtime PCR and presented as fold change over $\beta$-actin ( $\mathrm{n}=8$ mice/group). " $\mathrm{p}<0.05$ as compared to PMa from BALB/c mice. (B) IL-10 mRNA expression of PMa from control and LPS-injected mice was tested by real-time PCR and presented as fold change over $\beta$-actin ( $\mathrm{n}=8$ mice/group). " $\mathrm{p}<0.05$ as compared to PMa from $\mathrm{BALB} / \mathrm{c}$ mice.

Effect of pan-T cells on production of cytokines by macrophages in vitro. There were no differences in TNF- $\alpha$, IL- 6 and IL-10 levels in supernatants between PMa cultured alone and PMa co-cultured with pan-T cells before LPS stimulation. At $2 \mathrm{~h}$ after LPS stimulation, the levels of TNF- $\alpha$ and IL- 6 were higher in the supernatants from PMa cultured alone compared to co-cultured cells. There was no difference in IL-10 levels in the supernatants between PMa cultured alone and co-cultured cells at $2 \mathrm{~h}$ after LPS stimulation (Fig. 5).

Effect of pan-T cells on MKP-1 mRNA and protein expression in $P M a$ in vitro. PMa MKP-1 mRNA expression of PMa cultured with or without pan-T cells increased dramatically after LPS stimulation. As compared to PMa cultured alone, MKP-1 mRNA expression in PMa from co-cultured cells was higher at $30 \mathrm{~min}$ after LPS stimulation $(\mathrm{P}<0.05)$ (Fig. 6A).

MKP-1 protein expression of PMa cultured with or without pan-T cells increased after LPS stimulation. At $30 \mathrm{~min}$ after LPS stimulation, MKP-1 protein expression in PMa from co-cultured cells increased. At $60 \mathrm{~min}$ after LPS stimulation, MKP-1 protein expression in PMa from both groups reached the peak concentration and was higher in co-cultured cells than in PMa cultured alone $(\mathrm{P}<0.05)$. At $120 \mathrm{~min}$ after LPS stimulation, MKP-1 protein expression decreased, and there was no difference in MKP-1 expression between PMa cultured alone and co-cultured cells (Fig. 6B).

\section{Discussion}

The viewpoints on the control of innate immune cells by the adaptive immune system during sepsis remain controversial
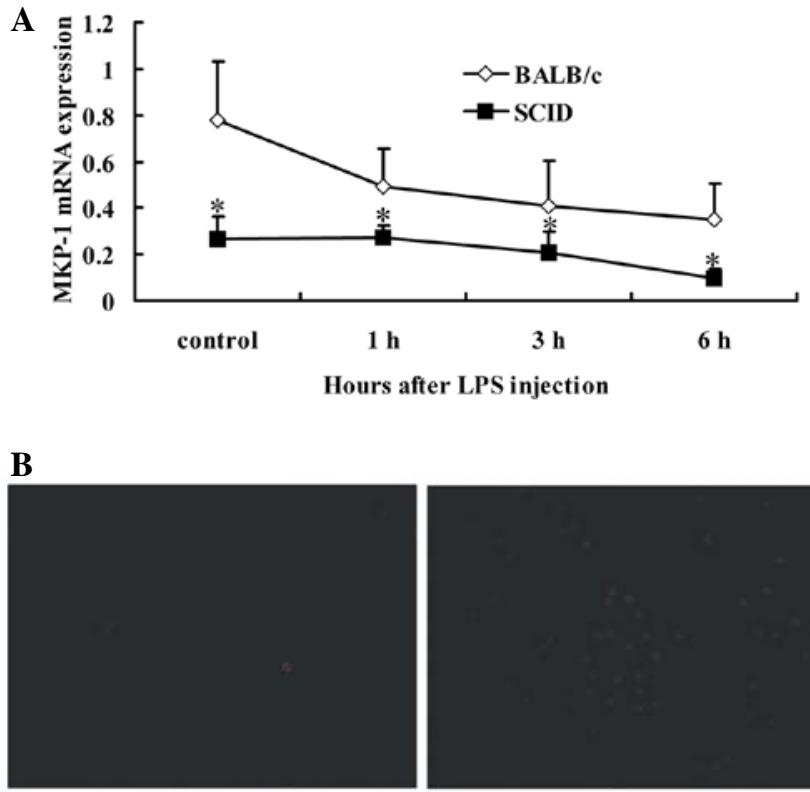

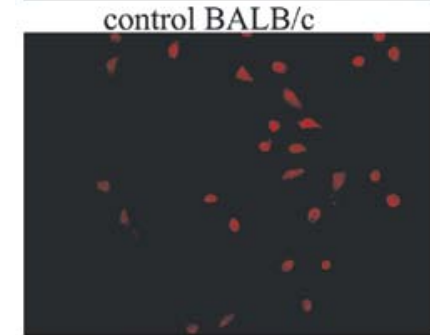

injected BALB/c

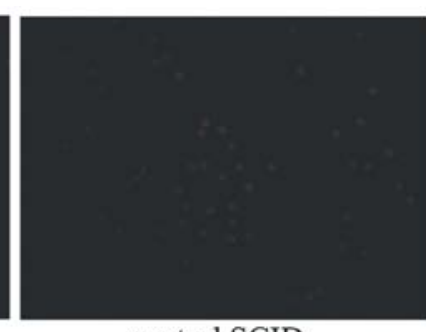

control SCID

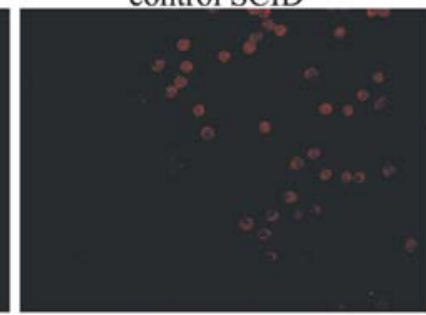

injected SCID
Figure 4. MKP-1 expression in PMa from BALB/c mice and SCID mice. (A) MKP-1 mRNA expression in PMa from control and LPS-injected mice was tested by real-time PCR and presented as fold change over $\beta$-actin ( $\mathrm{n}=8$ mice/ group). Lower MKP-1 expression in PMa from SCID mice as compared to PMa from BALB/c mice. " $\mathrm{p}<0.05$ as compared to PMa from BALB/c mice. (B) MKP-1 protein expression in PMa from BALB/c mice and SCID mice tested by immunofluorescence. At $1 \mathrm{~h}$ after LPS injection, MKP-1 expression was more pronounced in PMa from BALB/c mice compared with SCID mice.

(14-18). Research using mouse models of sepsis induced by cecal ligation and puncture (CLP) (19) produced different conclusions. Enoh et al (15) showed that $\mathrm{CD}^{+} \mathrm{T}$-cell depletion is not associated with alternations in survival, bacterial clearance and inflammation of mice with CLP-induced sepsis. Reim et al (17) showed that $\mathrm{T}$ cells contribute both to activation of innate immune cells and to amplification of the immune response against invading pathogens in mice with CLP-induced sepsis, and other septic models have yielded different viewpoints. van Schaik et al (14) showed that T cell-deficient mice exhibit markedly decreased disease severity compared to wild-type mice after $E$. coli injection and suggested that T cells may stimulate innate immune responses, resulting in enhancement of disease. The research on BALB/c mice and Rag-deficient mice with peritonitis induced by zymosan showed that lymphocytes do not have a significant impact on zymosan peritonitis in mice (16). Kim et al (9) showed that lymphocytes suppress activation of innate immune cells in Rag-1 knockout mice with poly (I: C)-induced SIRS. The shortage of septic models induced by CLP or E. coli suggests that the effect of interference of pathogen generations and spread of infection on inflammation caused by immune deficiency will bring difficulties for 


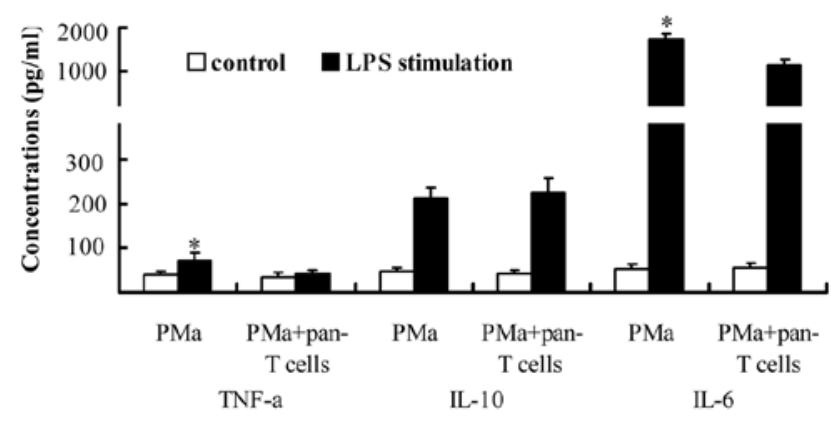

Figure 5. Increased TNF- $\alpha$ level in supernatants from PMa cultured alone as compared to supernatants from PMa co-cultured with pan-T cells after LPS stimulation. Concentrations of TNF- $\alpha$ and IL-10 in supernatants from PMa cultured alone and PMa co-cultured with pan-T cells at $2 \mathrm{~h}$ after LPS stimulation were tested by ELISA ( $\mathrm{n}=8$ samples/group). ${ }^{*} \mathrm{p}<0.05$ as compared to PMa co-cultured with pan-T cells.

the comparation of inflammation between wild-type and SCID mice with sepsis. Moreover, some studies in which non-infective stimulators, such as zymosan and poly (I: C), were used do not come to the same conclusion. Gram-negative bacterial infection is one of the common causes of sepsis in the clinic. Bacteria and endotoxin can act together, and endotoxin can act independently to promote sepsis. Endotoxin has such complex biological effects that it can trigger uncontrolled inflammation, immunological disorders, hypermetabolism status and multiple organ injury directly or indirectly (20). Therefore, LPS was used in this study to observe the effect of lymphocytes deficiency on innate immunity and inflammation.

In this study, survival rates, pathological sections of organs and levels of serum ALT and AST in BALB/c and SCID mice with endotoxemia showed that SCID mice had more severe organ injuries relative to $\mathrm{BALB} / \mathrm{c}$ mice in response to LPS. Neutrophil infiltration into organs and higher levels of inflammatory and anti-inflammatory cytokines in the serum of SCID mice indicated that SCID mice suffered and died from excessive inflammation when given the same dosage of LPS as $\mathrm{BALB} / \mathrm{c}$ mice (21). The augmented inflammation in SCID mice was caused by a loss of lymphocytes instead of pathogenic spread.

Macrophages are the main cells that produce inflammatory cytokines such as TNF- $\alpha$ and IL-6 (22). Higher levels of inflammatory cytokines in the serum of LPS-injected SCID mice compared to the serum of LPS-injected BALB/c mice suggested that macrophages from SCID mice should be more activated. Peritoneal fluid and PMa were used to study the local inflammation and activation of macrophages. When given the same dosage of LPS intraperitoneally, PMa from SCID mice secreted more inflammatory cytokines than PMa from BALB/c mice, and the basal TNF- $\alpha$ mRNA expression in resting PMa from SCID mice was higher than PMa from BALB/c mice. Both mice were on the same genetic background and were housed in specific pathogen-free setting, but they might come into contact with non-infective antigens in foods or other sources, which can activate their immune system. Thus, without lymphocytes, the activity of innate immune cells may be changed. The cytokine storm in SCID mice with endotoxemia implied that macrophage activation in SCID mice is partially similar to the excessive activation of macrophages in macrophage activation syndrome

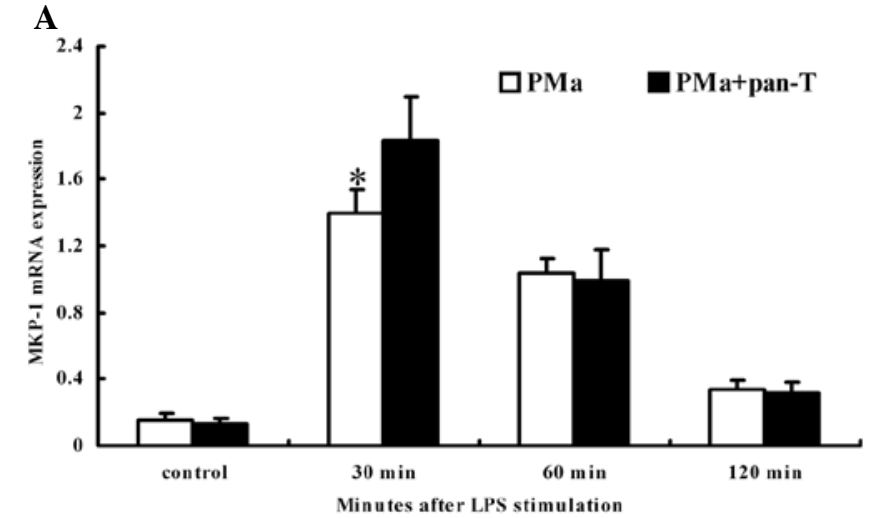

B
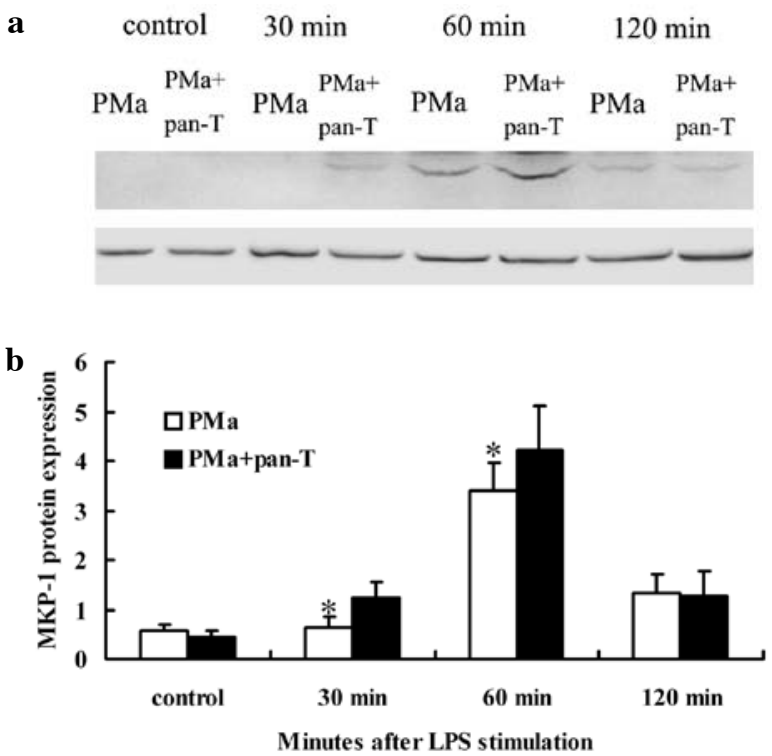

Figure 6. Decreased MKP-1 expression in PMa from PMa cultured alone as compared to PMa co-cultured with pan-T cells after LPS stimulation. (A) MKP-1 mRNA expression in PMa from PMa cultured alone and PMa cocultured with pan- $\mathrm{T}$ cells was tested by real-time PCR ( $\mathrm{n}=8$ samples/group). " $\mathrm{p}<0.05$ as compared to PMa co-cultured with pan-T cells. (B)a, MKP-1 protein expression in PMa from PMa cultured alone and PMa co-cultured with pan-T cells was tested by Western blotting, $\beta$-actin was used as internal control for protein loading ( $\mathrm{n}=5$ samples/group). $\mathrm{b},{ }^{*} \mathrm{p}<0.05$ as compared to PMa co-cultured with pan-T cells.

(MAS) (23). MAS in the clinic always has the molecular background of a defect in innate immunity (24), and in this study, lymphocyte deficiency also caused excessive activation of macrophages.

When faced with foreign pathogens, macrophages become activated through recognition of PAMPs by PRRs; a signal transduction cascade starts, and MAPK becomes activated. Activated MAPK translocates into the cellular nuclei and promotes the synthesis of cytokines, chemokines and other inflammatory mediators (25). There are three kinases of MAPK including ERK, JNK and p38; they all become activated through phosphorylation, so dephosphorylation by phosphatases is an important way to down-regulate MAPK activation. MKP are the most important phosphatases for MAPK, and MKP-1 is the most studied (26). MKP-1 inhibits activated MAPK, especially JNK and p38, by dephosphorylating MAPK tyrosine residues 
$(12,27,28)$. Then, the production of inflammatory cytokines can be reduced. Several TLR ligands can up-regulate MKP-1 expression including LPS, peptidoglycan, CpG DNA and poly (I: C) (13). After LPS stimulation, activation of p38 and JNK was prolonged in $\mathrm{MKP}-1^{-/}$macrophages, and production of TNF- $\alpha$ and IL- 6 was increased (13). Besides LPS, the other TLR ligands can also make MKP-1 ${ }^{-/}$macrophages produce more inflammatory cytokines. Therefore, MKP-1 is an essential negative factor of macrophage activation in Gram-positive and Gram-negative bacterial infection. Resting macrophages always express low MKP-1 expression, which allows for inflammatory responses after PAMP stimulation. After PAMP stimulation, MKP-1 mRNA expression increases by 10-100-fold between 15 to $60 \mathrm{~min}$ to suppress excessive inflammation (29).

Septic shock is one of the most important causes of death for patients with sepsis. Excessive production of inflammatory cytokines plays an important role in septic shock in pathophysiology. After LPS injection, MKP-1 ${ }^{-/}$mice produced more TNF- $\alpha$, IL-6 and IL-10 and were more prone to suffer from septic shock and MODS than wild-type mice. Dexamethasone, well-known for its anti-inflammatory property, can suppress inflammation during sepsis by up-regulating MKP-1 expression in macrophages (30). In this study, although MKP-1 mRNA expression in PMa from wild-type and SCID mice had no obvious increase after LPS injection, MKP-1 mRNA expression in PMa from $\mathrm{BALB} / \mathrm{c}$ mice was higher than in PMa from SCID mice at all of the tested time points. Therefore, lymphocyte deficiency was accompanied by decreased MKP-1 expression in macrophages from SCID mice explaining the excessive activation of macrophages and uncontrolled inflammation in SCID mice.

To clarify the direct inhibition of lymphocytes on macrophage activation and the role of MKP-1 in this inhibition, PMa and pan-T cells were isolated, and PMa were stimulated by LPS in the presence or absence of pan-T cells. The results showed that pan-T cells can inhibit the production of inflammatory cytokines by macrophages even at $2 \mathrm{~h}$ after LPS stimulation. MKP-1 is an early negative regulatory molecule of macrophages, and after LPS stimulation, MKP-1 mRNA and protein expression in PMa increased in both groups of cells and especially in co-cultured cells. Therefore, pan-T cells can up-regulate MKP-1 expression in macrophages and thus suppress the production of inflammatory cytokines.

The activity of MKP-1 can be regulated at several levels including transcriptional, protein stability and catalyze activity (31,32). MKP-1 mRNA is highly stable and the increase in MKP-1 protein correlates closely with the deactivation of JNK and p38 (33). MKP-1 mRNA and protein expression increased after LPS stimulation. Thus, the production of inflammatory cytokines was inhibited. In this study, the result showed that the regulation of MKP-1 expression by lymphocytes was at the transcriptional level.

Insufficient MKP-1 expression in macrophages can be responsible for the severe inflammation and bad prognosis of septic SCID mice. In SCID mice, the lymphocyte deficiency, especially the $\mathrm{T}$ cell deficiency, causes insufficient MKP-1 expression in macrophages, which was deleterious to the negative regulatory axis of macrophages activation. This can result in excessive activation of macrophages, cytokines storm and different clinical manifestations such as SIRS and MARS. MKP-1 plays an important role in the control of macrophage activation by the adaptive immune system. If this role can be further studied, it may provide insights on the mechanism and treatment of syndromes such as sepsis, SARS and IAHS.

\section{Acknowledgements}

This work was supported in part by grants from the National Nature Science Foundation of China. We thank the Academy of Medical Sciences of China for the mice. We also thank the Animal Care Facility Unit in Chongqing Children's Hospital, Chongqing Medical University for animal care.

\section{References}

1. Vincent JL, Martinez EO and Silva E: Evolving concepts in sepsis definitions. Crit Care Nurs Clin North Am 23: 29-39, 2011.

2. Filipovich AH: Hemophagocytic lymphohistiocytosis (HLH) and related disorders. Hematology Am Soc Hematol Educ Program 2009: 127-131, 2009.

3. Wong SS and Yuen KY: The management of coronavirus infections with particular reference to SARS. J Antimicrob Chemother 62: 437-441, 2008

4. To KK, Hung IF, Li IW, et al: Delayed clearance of viral load and marked cytokine activation in severe cases of pandemic H1N1 2009 influenza virus infection. Clin Infect Dis 50: 850-859, 2010.

5. Goncalves GM, Zamboni DS and Camara NO: The role of innate immunity in sepsitc acute kidney injuries. Shock (Suppl 1): 22-26, 2010.

6. Iwakiri D, Zhou L, Samanta M, et al: Epstein-Barr virus (EBV)encoded small RNA is related from EBV-infected cells and activates signaling from Toll-like receptors. J Exp Med 206: 2091-2099, 2009.

7. Frieman M, Heise M and Baric R: SARS coronavirus and innate immunity. Virus Res 133: 101-112, 2008.

8. Zhou J, Yang XQ, Fu Z, Zhao XD, Jiang LP, Wang LJ and Cui YX: Increase pathogenesis and inflammation of airways from respiratory syncytial virus infection in T cell deficient nude mice. Med Microbiol Immunol 197: 345-351, 2008.

9. Kim KD, Zhao J, Auh S, Yang X, Du P, Tang H and Fu YX: Adaptive immune cells temper initial innate responses. Nat Med 13: 1248-1252, 2007.

10. Keyse SM: Protein phosphatases and the regulation of mitogenactivated protein kinase signaling. Curr Opin Cell Biol 12: 146-150, 2000.

11. Li L, Chen SF and Liu Y: MAP kinase phosphatase-1, a critical negative regulator of the innate immune response. Int J Clin Exp Med 2: 48-67, 2009.

12. Hammer M, Mages J, Dietrich H, Servatius A, Howells N, Cato AC and Lang R: Dual specificity phosphatase1 (DUSP1) regulates a subset of LPS-induced genes and protects mice from lethal endotoxin shock. J Exp Med 203: 15-20, 2006.

13. Salojin KV, Owusu IB, Millerchip KA, Potter M, Platt KA and Oravecz T: Essential role of MAPK phosphatase-1 in the negative control of innate immune responses. J Immunol 176: 1899-1907, 2006.

14. van Schaik SM and Abbas AK: Role of T cells in a murine model of Escherichia coli sepsis. Eur J Immunol 37: 3101-3110, 2007.

15. Enoh VT, Lin SH, Etogo A, Lin CY and Sherwood ER: CD4 ${ }^{+}$ T-cell depletion is not associated with alterations in survival, bacterial clearance, and inflammation after cecal ligation and puncture. Shock 29: 56-64, 2008.

16. Kolaczkowska E, Barteczko M, Plytycz B and Arnold B: Role of lymphocytes in the course of murine zymosan-induced peritonitis. Inflamm Res 57: 272-278, 2009.

17. Reim D, Westenfelder K, Kaiser-Moore S, Schlautkotter S, Holzmann B and Weighardt $\mathrm{H}$ : Role of $\mathrm{T}$ cells for cytokine production and outcome in a model of acute septic peritonitis. Shock 31: 245-250, 2009.

18. Martignoni A, Tschöp J, Goetzman HS, Choi LG, Reid MD, Johannigman JA, Lentsch AB and Caldwell CC: CD4-expressing cells are early mediators of the innate immune system during sepsis. Shock 29: 591-597, 2008.

19. Remick DG, Newcomb DE, Bolgos GL and Call DR: Comparison of the mortality and inflammatory response of two models of sepsis: lipopolysaccharide vs. cecal ligation and puncture. Shock 13: $110-116,2000$. 
20. Davies B and Cohen J: Endotoxin removal devices for the treatment of sepsis and septic shock. Lancet Infect Dis 11: 65-71, 2011.

21. Luo C, Yang XQ,Li X,Liu W and Wang LJ: Comparation of inflammation between wild and severe combined immunodeficiency mice with endotoxemia induced by lipopolysaccharide. Xi Bao Yu Fen Zi Mian Yi Xue Za Zhi 27: 44-46, 2011 (In Chinese).

22. Khan S, Shin EM, Choi RJ, Jung YH, Kim J, Tosun A and Kim YS: Suppression of LPS-induced inflammatory and NF- $\kappa B$ responses by anomalin in RAW 264.7 macrophages. J Cell Biochem 112: 2179-2188, 2011.

23. Billiau AD, Roskams T, Van Damme-Lombaerts R, Matthys $P$ and Wouters C: Macrophage activation syndrome: characteristic findings on liver biopsy illustrating the key role of activated, IFN-gamma-producing lymphocytes and IL-6 and TNF-alphaproducing macrophages. Blood 105: 1648-1651, 2005.

24. Villanueva J, Lee S, Giannini EH, Graham TB, Passo MH Filipovich A and Grom AA: Natural killer cell dysfunction is a distinguishing feature of systemic onset juvenile rheumatoid arthritis and macrophage activation syndrome. Arthritis Res Ther 7: R30-R37, 2005

25. Chi H, Barry SP, Roth RJ, Wu JJ, Jones EA, Bennett AM and Flavell RA: Dynamic regulation of pro- and anti-inflammatory cytokines by MAPK phosphatase 1 (MKP-1) in innate immune responses. Proc Natl Acad Sci USA 103: 2274-2279, 2006.

26. Jacob A, Rajan D, Pathickal B, Balouch I, Hartman A, Wu R, Zhou $\mathrm{M}$ and Wang P: The inhibitory effect of ghrelin on sepsisinduced inflammation is mediated by the MAPK phosphatase-1. Int J Mol Med 25: 159-164, 2010.
27. Franklin CC and Kraft AS: Conditional expression of the mitogen-activated protein kinase (MAPK) phophatase MKP-1 preferentially inhibits p38 MAPK and stress-activated protein kinase in U937 cells. J Biol Chem 272: 16917-16923, 1997.

28. Zhao Q, Wang X, Nelin LD, et al: MAP kinase phosphatase 1 controls innate immune response and suppresses endotoxic shock. J Exp Med 203: 131-140, 2006.

29. Wang X and Liu Y: Regulation of innate immune response by MAP kinase phosphatase-1. Cell Signal 19: 1372-1382, 2007.

30. Shipp LE, Lee JV, Yu CY, Pufall M, Zhang P, Scott DK and Wang JC: Transcriptional regulation of human dual specificity protein phosphatase 1 (DUSP1) gene by glucocorticoids. PLoS One 5: e13754, 2010

31. Sohaskey ML and Ferrell JE Jr: Activation of p42 mitogenactivated protein kinase(MAPK), but not c-Jun NH(2)-terminal kinase, induces phosphorylation and stabilization of MAPK phosphatase XCL 100 in Xenopus oocytes. Mol Biol Cell 13: 454-468, 2002

32. Cao W, Bao C, Padalko E and Lowenstein CJ: Acetylation of mitogen-activated protein kinase phosphatase-1 inhibits Toll-like receptor signaling. J Exp Med 205: 1491-1503, 2008.

33. Chen P, Li J, Barnes J, Kokkonen GC, Lee JC and Liu Y: Restraint of proinflammatory cytokine biosynthesis by mitogen-activated protein kinase phosphatase-1 in lipopolysaccharide stimulated macrophages. J Immunol 169: 6408-6416, 2002. 